\title{
Regresión espontánea tras nefrectomía de carcinoma renal metastásico. Aportación de un caso con confirmación histológica y revisión de la literatura
}

\author{
J. I. Rodríguez, P. Soria, A. Sola, M. López, M. Bustos, A. Rodríguez, O. Alonso, A. Nieto, \\ B. García, J. L. Rodríguez
}

\section{Resumen}

El carcinoma renal suele manifestarse clínicamente de forma tardía, por lo que aproximadamente un tercio de los pacientes presentan metástasis a distancia en el momento del diagnóstico. En estos casos, la supervivencia disminuye drásticamente, situándose en una media de 12 meses. Se han descrito algunos casos de regresión espontánea (RE) en este tumor, que varían desde regresiones completas y duraderas hasta parciales y temporales. La RE se presenta entre el 0,4 y el $4 \%$ de los pacientes con carcinoma renal mestastásico (CRM). A pesar de estar descritos muchos factores relacionados con este raro y favorable suceso, no se conoce su verdadero origen y no es, por tanto, un evento predecible. Presentamos un caso de RE tras nefrectomía en un paciente con carcinoma renal, que presentaba metástasis pulmonares confirmadas histológicamente y recidiva local.

Palabras clave: Carcinoma Renal. Regresión Espontánea. Nefrectomía.

Oncología, 2006; 29 (6):263-273

\section{Summary}

The clinical manifestations of renal cell carcinoma are often late, so that approximately one third of the patients have metastatic disease at the time of diagnosis. In this case, the survival rate diminishes dramatically, with an average survival time of 12 months. However, some cases of spontaneous regression have been reported for this kind of tumor, either complete and permanent or partial and temporary. Spontaneous regression occurs in $0.4-4.0 \%$ of the patients with metastatic renal cell carcinoma. Many factors have been proposed as related with this rare but favorable event, but until now the origin remains unknown and no prediction can be made. We present a case of renal cell carcinoma with histologic confirmation of lung metastases and local relapse showing a spontaneous regression after nephrectomy.

Key words: Renal cell carcinoma. Spontaneous regression. Nephrectomy. 


\section{Introducción}

El Carcinoma Renal (CR) representa el $2 \%$ de todos los tumores malignos ${ }^{1}$. Habitualmente se presenta entre la $5^{\text {a }}$ y la $7^{\text {a }}$ décadas de la vida ${ }^{1}$ y su clínica -hematuria, dolor lumbar, masa palpable- suele manifestarse de forma tardía en el curso de la enfermedad $^{1,2}$. Por este motivo, entre el 25 y el $40 \%$ de los pacientes presentan metástasis a distancia en el momento del diagnóstico ${ }^{1,3-5}$, las más frecuentes en pulmón, hígado, hueso y sistema nervioso central $^{3}$. Es un tumor impredecible que nunca se puede considerar curado $^{3}$, y el $85 \%$ de las recaídas aparecen en los 3 primeros años ${ }^{1}$.

El tratamiento de elección en los estadios localizados es la nefrectomía (NF) con una supervivencia a los 5 años en estadios I del 80-100\% ${ }^{6}$. Los pacientes con ganglios positivos tienen un peor pronóstico, falleciendo el $80-90 \%$ de los mismos en el primer año ${ }^{3}$. En adyuvancia tras la cirugía, la radioterapia (RT) no aumenta la supervivencia pero mejora el control local en indicaciones concre$\operatorname{tas}^{2}$. A pesar de esto, se asume que es un tumor resistente de forma intrínseca a la irradiación ${ }^{7}$. Tampoco se ha conseguido incrementar la supervivencia con quimioterapia $(\mathrm{QT})^{1}$, considerándose un tumor quimiorresistente ${ }^{1,7}$ entre otros factores por la alta expresión de resistencia a múltiples drogas y el alto contenido de glutation de las células tumorales ${ }^{4}$.

El Carcinoma Renal Metastásico (CRM) tiene una supervivencia media de entre 8 y 18 meses $^{1,4,8,9}$, a los 2 años la supervivencia cae al $10 \%$ y a los 5 años los supervivientes no superan el 5\%, .

El tratamiento del CRM en la actualidad incluye desde la NF, tradicionalmente de elección ${ }^{1-5,10,11}$ con cirugía de metástasis única resecable (en casos muy seleccionados $)^{5}$ hasta cuidados paliativos y medidas de soporte exclusivamente. La inmunoterapia con Interferón (IFN) o Interleukina 2 (IL-2) con o sin IFN representa en la actualidad el tratamiento sistémico más aceptado ${ }^{12}$, a pesar de su elevada toxici$\operatorname{dad}^{1,4,7,9}$ y de no alterar la supervivencia ${ }^{1}$. Las respuestas a IFN ( $\alpha$-2a, $\alpha$-2b y $\gamma-1 b)$ publicadas varían entre el 10 y el $20 \%$, mientras que en el caso de la IL-2 se sitúan en el 10-15\%, un 5-10\% de las cuales son respuestas completas, algunas con duraciones superiores a los 2 años 1, 3, 4, 7, 9. La Quimioterapia en el CRM se sitúa en el ámbito de ensayos clínicos con agentes como la Gemcitabina ${ }^{12}$ y más recientemente el Bevacizumab como segunda línea ${ }^{13}$.

En el CRM están descritos casos de regresión espontánea (RE) de la enfermedad ${ }^{1,3,10,11,14-42}$, con desaparición de algunas o todas las metástasis del paciente, generalmente tras $\mathrm{NF}^{25}, 27,28,30,33,34,38,39,41$, aunque también tras radioterapia local paliativa sobre el tumor primario ${ }^{11,43}$.Según distintos autores la frecuencia de aparición de RE en el CRM varía desde el 0,4 al $4 \%{ }^{1,3}$.

En este artículo presentamos el caso de un paciente remitido a nuestro servicio para someterse a tratamiento radioterápico por un adenocarcinoma de próstata localizado, al que en el estudio de extensión se diagnostica un hipernefroma con metástasis pulmonares.

\section{Caso clínico}

Varón de 73 años en su primera visita a nuestro Servicio, con antecedentes de diabetes mellitus, cardiopatía isquémica, hábito tabáquico hasta 1993, sin alergias conocidas a medicamentos. En Julio de 2001, tras varios meses de molestias rectales, se objetiva en analítica de rutina una cifra de PSA de 16.68 ng/ml. Remitido desde Atención Primaria a Urología, es diagnosticado mediante biopsia de Adenocarcinoma de Próstata en lóbulo derecho, Gleason $3+3\left(\mathrm{~T}_{1 \mathrm{c}} \mathrm{N}_{\mathrm{x}} \mathrm{M}_{\mathrm{x}}\right.$, Estadio II).

Tras instaurarse bloqueo androgénico completo (BAC) con Bicalutamida y Leuprorelina es remitido a nuestro Servicio, doride se solicita TC tóraco-abdominal para completar estadiaje. Pendiente del resultado de dicha prueba debido a la demora en su realización y teniendo en cuenta la edad el paciente y el estadio de la enfermedad, se inicia el tratamiento RT. Se programa una dosis de 70 Gy sobre próstata y vesículas seminales, usando fotones de 18 $\mathrm{MV}$, técnica de 4 campos en caja y fraccionamiento de 2 Gy/día, 5 días por semana.

Antes de la finalización del tratamiento se recibe la TC (11/10/01), en la que se objetiva una masa sólida mediastínica de $3 \mathrm{~cm}$, paraesofágica izquierda, superior a cayado aórtico, no pudiendo diferenciarse si se trata de una masa adenopática o una neoplasia primitiva (Fig. 1). Además se visualiza una masa sólida renal derecha, de aproximadamente $7 \mathrm{~cm}$ de diámetro, con necrosis central, sugerente de hiper- 


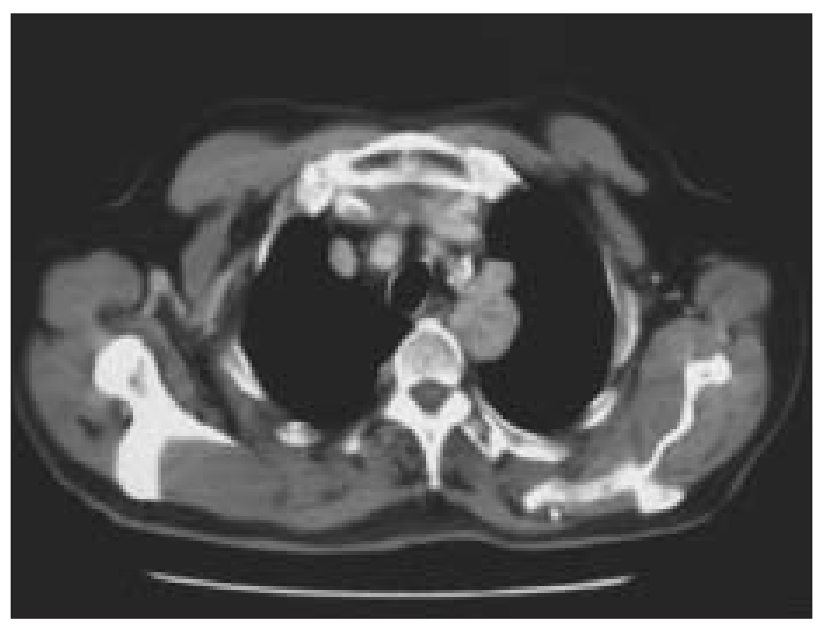

Figura 1.

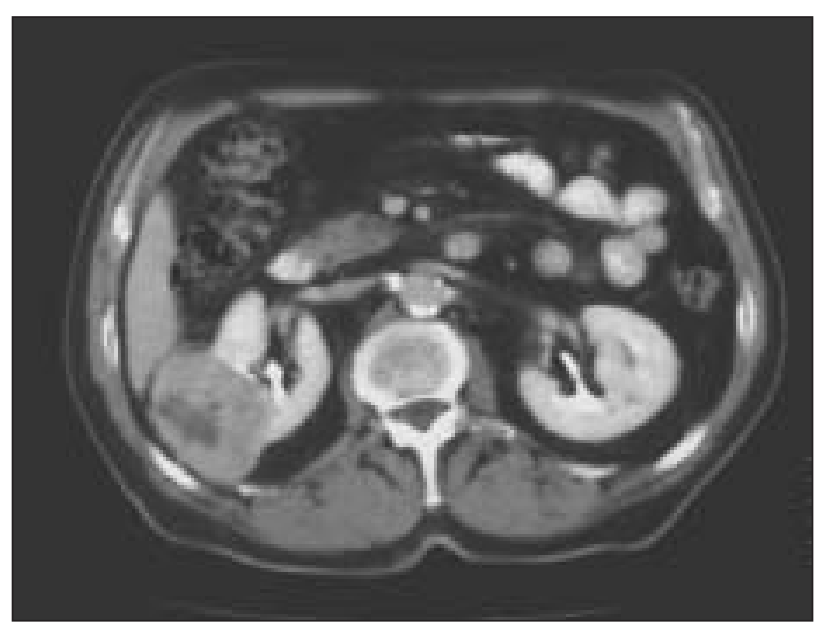

Figura 2.

nefroma (Fig. 2). No se objetivan alteraciones vasculares, adenopatías retroperitoneales ni alteraciones significativas en la pelvis.

Tras el resultado de la TC, se decide continuar la irradiación sobre la próstata, finalizándose el tratamiento el 26/11/01. Mientras tanto, y dado que la masa renal no planteaba dudas diagnósticas, se opta por el abordaje de la lesión torácica.

El 15/11/01 se lleva a cabo una mediastinoscopia con toma de biopsias, con resultado histológico de carcinoma de células claras, compatible con metástasis de Carcinoma Renal.

De acuerdo con Oncología Médica y Urología, el 21/12/01 se realiza la Nefrectomía (NF), con resultado histológico definitivo de carcinoma de células renales grado II-III, que invade tejido adiposo perirrenal $\left(\mathrm{T}_{3 \mathrm{a}} \mathrm{N}_{\mathrm{x}} \mathrm{M}_{1}\right.$, Estadio IV).

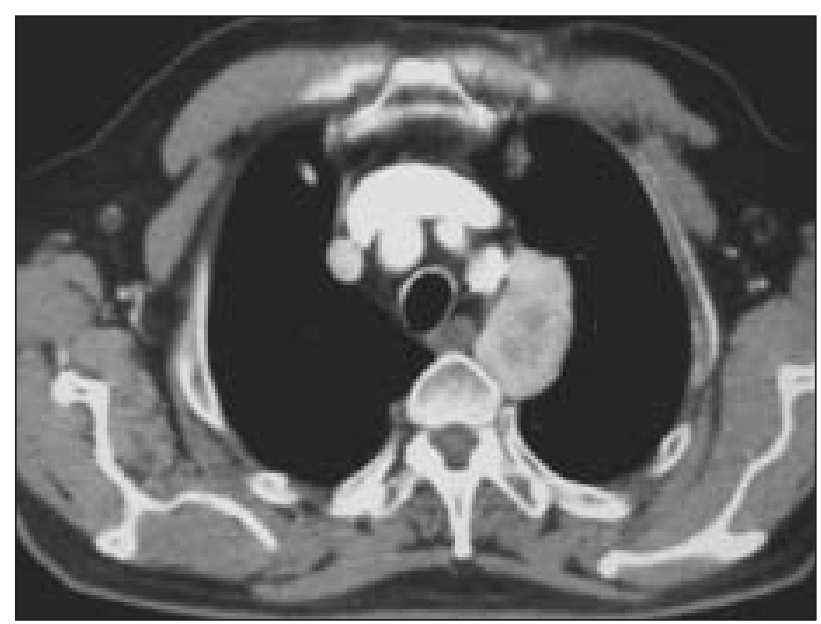

Figura 3.

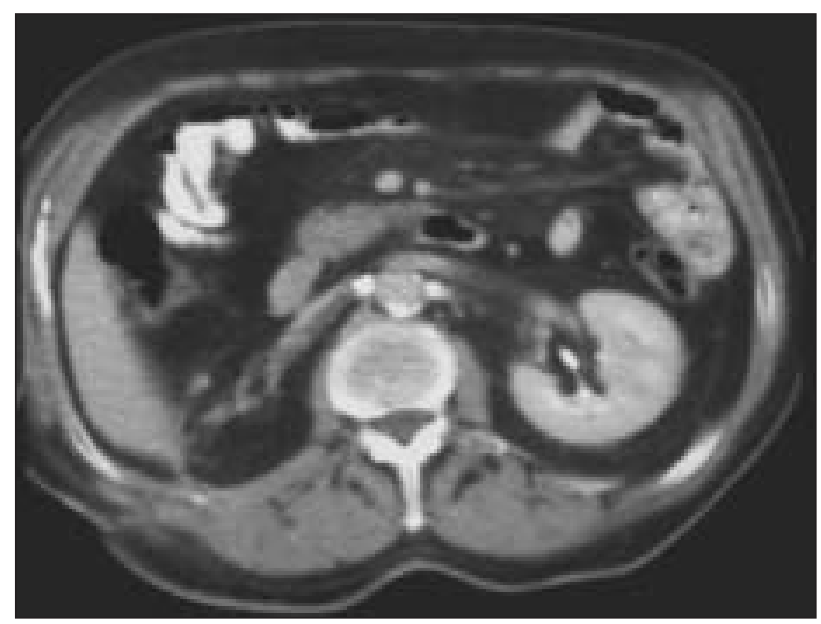

Figura 4.

Ante la posibilidad de cirugía sobre la metástasis mediastínica, se realiza nueva TC (30/01/02), en la que se constata la presencia de la masa pulmonar, con un tamaño aproximado de 5 x 3 x $4 \mathrm{~cm}$ (Fig. 3) que infiltra región mediastínica posterior, sin plano de clivaje con estructuras vasculares supraaórticas ni con el cayado aórtico, encontrándose muy próxima a tercio superior esofágico. A nivel abdominal se visualiza la NF derecha sin signos de recidiva ni resto tumoral (Fig. 4).

El 27/02/02 se realiza toracotomía exploradora, con el hallazgo de tumor mediastínico de $5 \mathrm{~cm}$ que invade parénquima pulmonar y cara superior del cayado aórtico a nivel de una placa de arterioesclerosis que impide el clampaje lateral, por lo que se considera irresecable. Se toma biopsia y se confirma el diagnóstico. 


\section{J. I. Rodríguez y cols.}

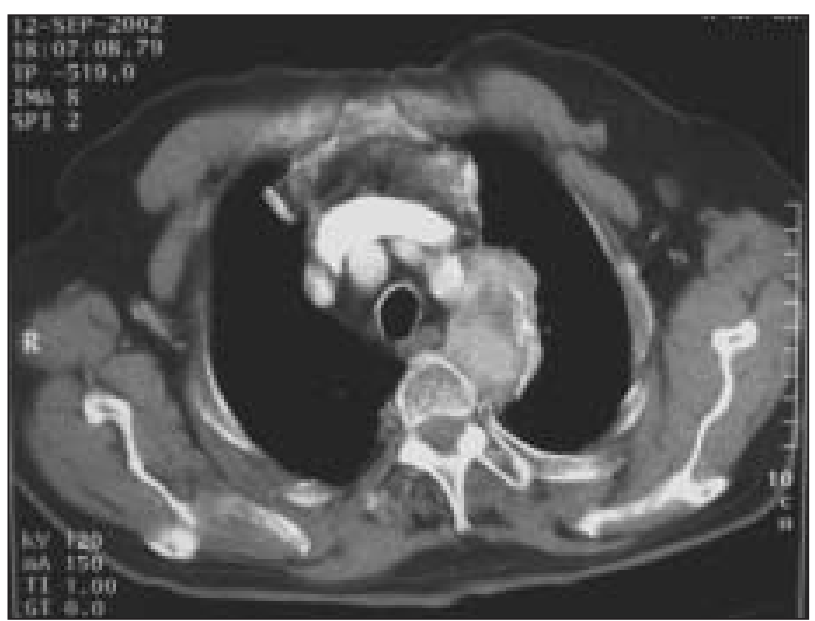

Figura 5.

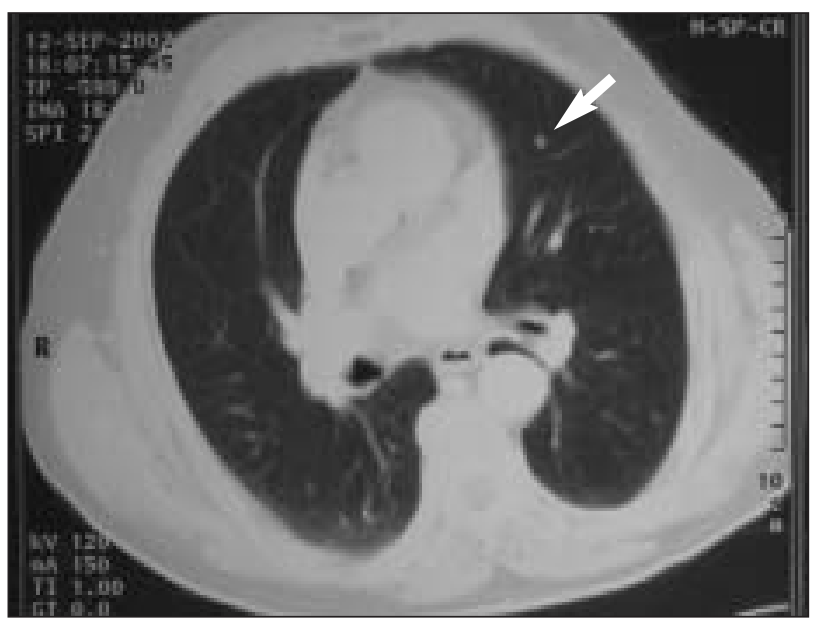

Figura 6.

Se plantea la posibilidad de tratamiento radioterápico sobre la lesión mediastínica, seguida o no de inmunoterapia, pero el paciente no acepta tal posibilidad y considerando su buen estado general, se adopta una actitud expectante.

El paciente continúa con $\mathrm{BAC}$ y tratamiento sintomático. En Junio de 2002 recibe tratamiento con Acetato de Megestrol por "sofocos" secundarios al BAC, que se interrumpe un mes después al suspenderse el BAC. En Septiembre de 2002 se realiza una gammagrafía ósea, que no evidencia depósitos patológicos del trazador, así como una determinación del PSA $(0.10 \mathrm{ng} / \mathrm{ml})$ y una nueva TC. En dicha TC (12/09/02) se aprecia masa en mediastino posterior, por encima del cayado aórtico, de $6 \times 4 \times 4 \mathrm{~cm}$. (Fig. 5) que infiltra la arteria subclavia izquierda, la aorta y el pulmón izquier-

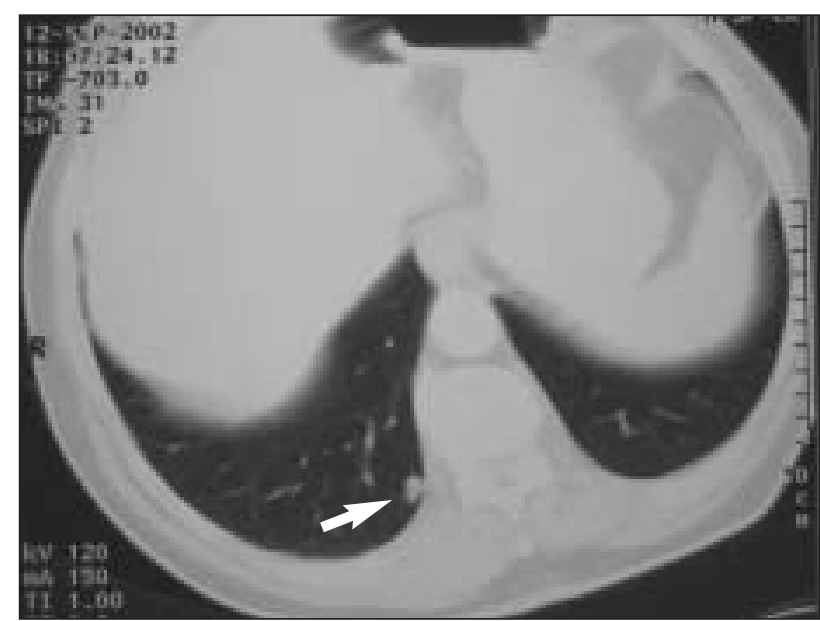

Figura 7.

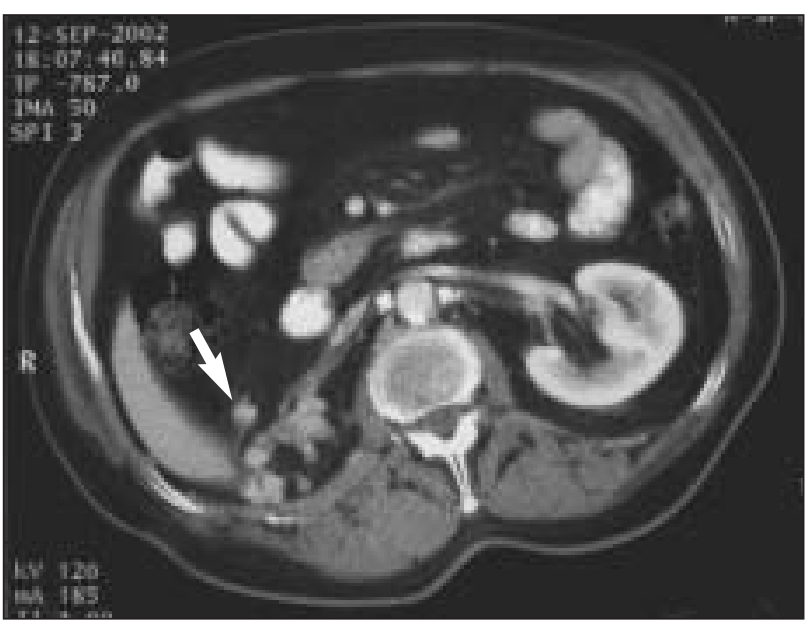

Figura 8 .

do, así como imágenes nodulares en língula (Fig. 6 , flecha) y lóbulo inferior derecho adyacente a la columna vertebral (Fig. 7, flecha), sugerentes de metástasis pulmonares. En el abdomen se visualizan múltiples imágenes nodulares en fosa renal derecha (Fig. 8, flecha) sugerentes de recidiva, con probable infiltración del músculo psoas ipsilateral.

Por lo tanto, y como conclusión tras el último TC ( 9 meses después de la NF) el paciente presenta una franca progresión de su enfermedad, con nuevas metástasis además de recidiva local.

En octubre de 2002 ingresa por sospecha clínica de tromboembolismo pulmonar, sin confirmación gammagráfica. Tras el alta es visto de forma ambulatoria en varias ocasiones, manifestando una mejoría progresiva de su estado general. 


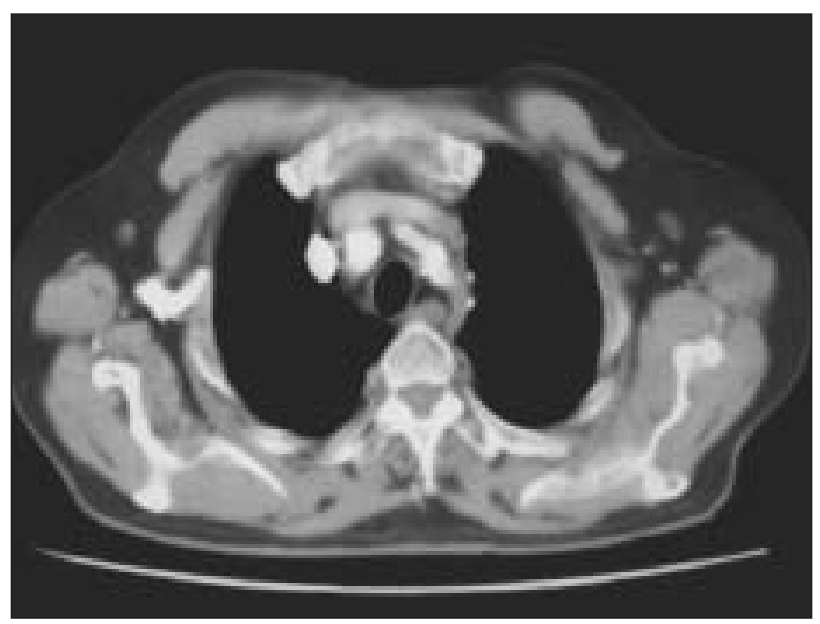

Figura 9.

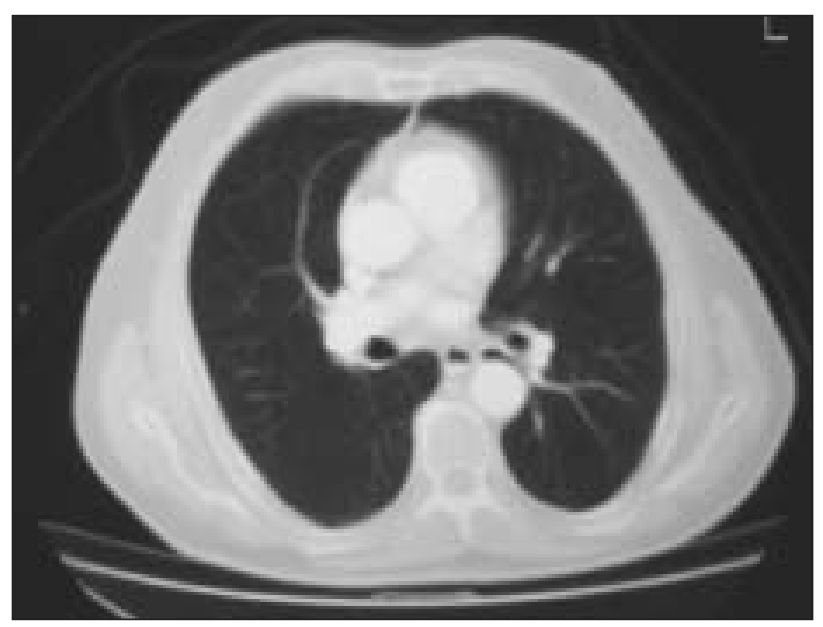

Figura 10.

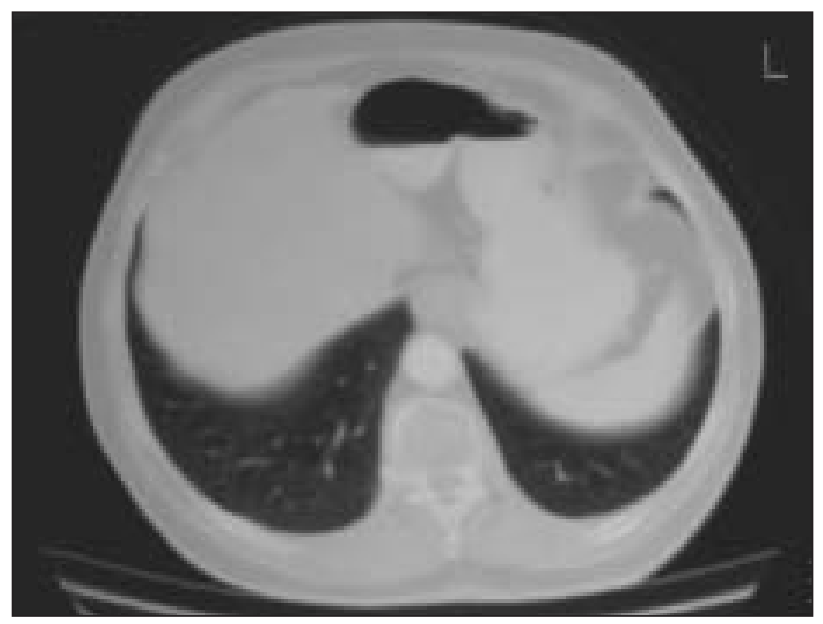

Figura 11.

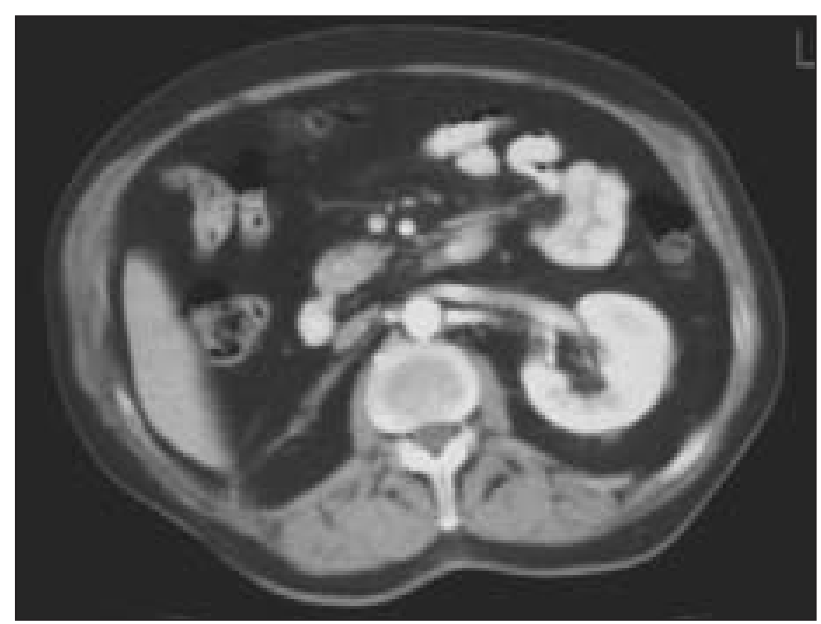

Figura 12.

\section{CUADRO I}

\section{Resumen caso clínico}

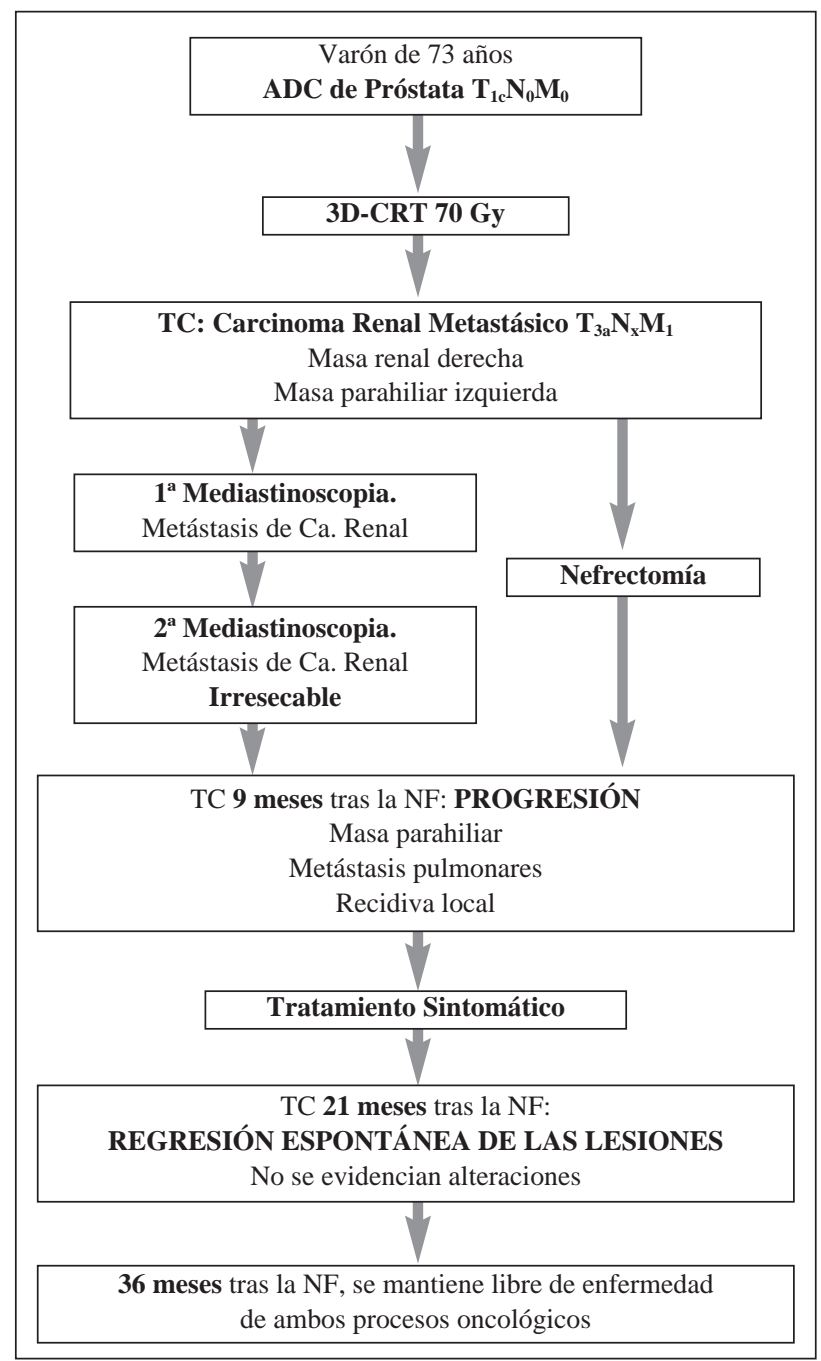


En septiembre de 2003, 21 meses tras la NF y encontrándose el paciente asintomático, se realiza TC torácica y abdominal (16/09/03), que muestra únicamente varias adenopatías axilares menores de $1 \mathrm{~cm}$ y la NF derecha, sin evidencia de otras lesiones -Respuesta radiológica completa- (Figs. 9-12).

En la actualidad (36 meses tras NF) dos TC posteriores muestran la persistencia de dicha respuesta y el paciente sigue asintomático. El tumor prostático continúa en respuesta completa, con nadir de PSA de $0,10 \mathrm{ng} / \mathrm{ml}$ y sin alteraciones en la gammagrafía ósea.

\section{Discusión}

\section{Regresión espontánea}

En 1964 Bloom y Payne ${ }^{44}$ definieron el concepto de RE como "la desaparición parcial o completa de un tumor maligno en ausencia de todo tratamiento, o en presencia de un tratamiento que es considerado inadecuado para esperar una influencia significativa en la enfermedad neoplásica, no debiendo considerarse RE sinónimo de curación".

Según Everson y Cole ${ }^{45}$, la RE puede clasificarse en 6 tipos diferentes: (1) RE del tumor primario; (2) RE de metástasis con confirmación histológica; (3) RE de metástasis sin confirmación histológica; (4) $\mathrm{RE}$ de presumibles metástasis (sólo con criterio radiológico); (5) Estabilización prolongada; y (6) Retraso en la aparición de metástasis o recurrencia.

La RE de un CRM es un evento ya conocido aunque infrecuente. Desde que en 1928 Bumpus describiera la primera RE de un $\mathrm{CRM}^{10}$, son múltiples los casos de este favorable acontecimiento publicados en la literatura.

El CR es el tumor con mayor frecuencia de RE, seguido del melanoma, el neuroblastoma y el coriocarcinoma $^{3,11}$. Como se ha dicho, la frecuencia de aparición de una RE en el CRM se sitúa entre el 0,4 y el $4 \%$ de los casos, aunque para otros autores esta frecuencia sería menor del 1\% ${ }^{1,3}$. El 70\% de los casos de RE en CRM son varones ${ }^{3,10}$.

El pulmón es el órgano que con mayor frecuencia se ve afectado por metástasis en el $\mathrm{CR}^{44}$. Además de las otras localizaciones ya mencionadas (hueso, SNC, hígado) están descritas otras más raras, como las metástasis intratiroideas ${ }^{46} \mathrm{o}$ las metástasis endo- bronquiales, que representan el 5\% de todas las metástasis pulmonares ${ }^{14}$. El papel de la cirugía está bien definido en el tratamiento de las metástasis pulmonares ${ }^{47}$ y en las de $\mathrm{SNC}^{48}$.

La localización de las metástasis que experimentan la RE es diversa, siendo la más frecuente el pulmón ${ }^{1,3,10,11,17, ~ 24, ~ 27, ~ 29-32, ~ 34, ~ 37, ~ 39, ~ 40,43, ~ 49, ~ s e g u i d a ~ d e ~}$ hueso $^{50}$, sistema nervioso central ${ }^{19,21,31}$, intestino, hígado $^{41,51}$, piel $^{37}$, coroides $^{20}$, páncreas ${ }^{52}$, tiroides y pleura $^{38}$.

Así mismo la evolución de la RE en el CRM es muy variable, habiéndose publicado supervivencias libres de enfermedad de más de 20 años ${ }^{3}$, regresión de determinadas metástasis y progresión de otras, así como recaídas o aparición de otras metástasis a medio plazo.

Generalmente la RE de un CRM suele producirse tras $\mathrm{NF}^{25}, 27,28,30,33,34,38,39,41$, aunque también se han descrito casos tras radioterapia local paliativa sobre el tumor primario ${ }^{11,43}$ y regresiones tras tratamiento con IFN exclusivamente. El periodo comprendido entre la realización de la NF y la aparición de una RE es muy variable, pudiendo abarcar desde varias semanas hasta 18 meses $^{10}$.

En la literatura revisada, los factores relacionados con la RE del CRM son los siguientes: (1) Pacientes que han presentado una RE sin haber sido sometidos a ningún acto médico ${ }^{3}$ y pacientes incluidos en brazo placebo de ensayos clínicos fase III $^{1,7}$; (2) radioterapia paliativa sobre el tumor primario ${ }^{11}$; (3) NF con o sin cirugía de metástasis $3,5,10,11,20,25,27,28$, $30,33,34,38,39,41$; (4) inmunoterapia con IFN $(\alpha-2 \mathrm{a}, \alpha-$ 2b y $\gamma$-1b) o IL-2 con o sin $\mathrm{IFN}^{3,8-10,21}$; (5) cambios hormonales y tratamientos con Medroxiprogesterona y Tamoxifeno ${ }^{3,8-10,21}$; (6) factores de crecimiento celular, citokinas (TNF- $\alpha$, TNF- $\beta$, IFN- $\gamma$, IL- 6 , receptores de IL-2) VSG, PCR, Ferritina y otros reactantes de fase aguda ${ }^{7,11,20}$ y (7) otros factores relacionados, como heridas purulentas o fístulas, inmunoestimulación con BCG, inmunomodulación mediante transplante alogénico de precursores no-mieloablativo, y la vitamina $\mathrm{E}^{7,10}$.

Stephenson et al. en 1971 demostraron al menos uno de estos tres factores -infección, cambios hormonales y alteración del estado inmune- en 184 (82\%) de 224 casos de CRM con $\mathrm{RE}^{36}$. Otros autores han descrito procesos de "autocuración" del primario, tales como fenómenos de cicatrización, aislamiento o "encapsulamiento" del tumor, y enveje- 
cimiento celular ${ }^{3}$, así como RE asociados a traumas, como la cirugía.

La mayor frecuencia de RE en las metástasis pulmonares podría deberse a la naturaleza del parénquima pulmonar, especialmente rico en macrófagos, linfocitos e inmunoglobulina $\mathrm{A}^{3}$. En ensayos randomizados las metástasis en órgano único (sobre todo en pulmón) predicen una mejor respuesta a la inmunoterapia combinada ${ }^{1}$. En algunos casos publicados hay simultáneamente RE de metástasis pulmonares con progresión de metástasis de otras localizaciones $^{3}$. En sólo un $20 \%$ de los casos de RE las metástasis pulmonares tenían confirmación histológica de su origen ${ }^{6}$, con lo que el porcentaje total de RE podría ser menor si se tiene en cuenta los posibles diagnósticos diferenciales con sarcoidosis, enfermedades del colágeno, infecciones fúngicas e incluso infartos pulmonares ${ }^{6}$.

\section{Papel de la NF en el CRM}

No está justificada la realización de una NF en pacientes asintomáticos con el único propósito de desencadenar una $\mathrm{RE}^{1-3}$, dada la baja frecuencia de este evento y la morbi-mortalidad de la NF. En este tipo de pacientes -oncológicos y con enfermedad metastásica- la mortalidad de la NF oscila del 2 al $15 \%$, aunque la mayoría de los autores declaran un $5 \%{ }^{3}$. En estos casos la NF se reserva para el control del dolor local, hemorragias, hipertensión arterial o hipercalcemia resistentes al tratamiento médico habitual $^{2}$. Sin embargo ha podido demostrarse que la NF aumenta la respuesta al tratamiento inmunológi$\mathrm{co}^{2}$. Tras la NF, la inmunoterapia con IFN con o sin IL-2 aumenta el porcentaje de regresiones hasta un $20 \%$ en algunos trabajos ${ }^{3}$, aunque para otros autores ${ }^{11}$ no está demostrado que la NF aumente la respuesta a tratamientos sistémicos. Dos estudios randomizados de EORTC y $\mathrm{SWOG}^{2}$ comparan los resultados del tratamiento exclusivo con IFN frente al tratamiento combinado de NF más IFN, mostrándose superior la asociación de los dos tratamientos (mediana de supervivencia de 7 vs. 17 meses y 8 vs. 11 meses respectivamente). En casos de metástasis única, tras nefrectomía y tratamiento de la metástasis se han alcanzado supervivencias a 5 y 10 años de hasta un $40 \%$ y un $15 \%$ respectivamente . $^{8}$

Las indicaciones para la realización de una NF en un paciente con CRM, en caso de fracaso del trata- miento médico, son las siguientes ${ }^{1-3}$ : (1) Eliminar una fuente de fiebre y toxicidad por el tumor primario; (2) revertir una hepatopatía; (3) resolución de anemia o eritrocitosis por superproducción de eritropoyetina; (4) tratamiento del dolor ocasionado por el tumor primario; (5) tratamiento de la HTA; (6) tratamiento de hematuria; (6) corregir la hipercalcemia; y (7) eliminar una fuente de parathormona-like.

Respecto al mecanismo por el cual la NF podría precipitar una RE de las metástasis, existen varias teorías. La primera de ellas plantea que durante la cirugía se produciría una diseminación de células tumorales en el torrente sanguíneo o linfático, que actuarían como carga antigénica tumoral, estimulando una fuerte respuesta inmune por parte del huésped $^{3}$. Otra teoría apunta a la reducción de masa tumoral o "debulky" como resultado de la NF; la carga antigénica del tumor primario sería demasiado grande para que el sistema inmune fuera capaz de reaccionar, la NF produciría una reducción de la mayor parte de carga antigénica, mejorando la acción efectora del sistema inmune ${ }^{3,11}$. Una última explicación sería la reducción tras la NF de los niveles de factores implicados en la angiogénesis (b-FGF, VEGF), que se encuentran aumentados en el $\mathrm{CRM}^{20}$.

\section{Hormonoterapia en el CRM}

Durante muchos años el CR fue considerado un tumor hormonodependiente. Entre los hallazgos que apoyaban esta teoría se encuentra la inducción con estrógenos de tumores renales en ratones ${ }^{8}$, el hecho de que la incidencia del CR por sexos esté equilibrada en menores de 45 años y que tras la menopausia sea de 2 a 3 veces superior en hombres ${ }^{1,3}$, el descubrimiento de receptores hormonales (estradiol, progesterona y dihidrotestosterona) en células del tumor $^{8}$, y la aparición de respuestas a algunos tratamientos hormonales. Estas respuestas se han producido con tratamientos con andrógenos y progestágenos (respuestas entre el 10 y el $16 \%)^{3,9}$ y con Tamoxifeno ${ }^{3}$. Sin embargo, en estudios posteriores, dichas respuestas no han podido ser reproducidas y los receptores celulares encontrados, además de ser mucho menos cuantiosos que, por ejemplo, en tumores mamarios, no poseen su importancia ni influencia en las respuestas a tratamientos ${ }^{3,8}$. De he- 
cho, no hay correlación entre la expresión de receptores y la aparición de recidiva local o la supervivencia $^{8}$.

Nuestro paciente recibió tratamiento con acetato de megestrol (40 mg/día) durante 2 meses por presentar sofocos secundarios a la deprivación androgénica. A este respecto Kjaer et $\mathrm{al}^{8}$ en 1988 publicaron una revisión sobre el papel de la medroxiprogesterona en el CR, tanto sobre su uso en adyuvancia tras NF en estadios iniciales, como su uso en el tratamiento del CRM. Tras revisar un periodo de 30 años observaron que en CRM las respuestas descritas en estudios anteriores (1-2\%) son respuestas parciales y de corta duración, independientes de la dosis y del esquema de tratamiento empleado, sin impacto en la supervivencia libre de recidiva ni en la supervivencia global. En la actualidad no está indicado el uso de medroxiprogesterona como tratamiento antineoplásico del CRM, reservándose su utilización para casos de anorexia asociada al cáncer $^{9,11,12}$, mejoría del estado general ${ }^{2,9}{ }^{11}$ y para el tratamiento de los sofocos secundarios a tratamientos hormonales ${ }^{53}$.

\section{Carcinoma renal e Inmunidad}

El IFN- $\alpha$ y la IL-2 son componentes pleiotrópicos con efectos específicos sobre numerosas poblaciones leucocitarias ${ }^{4}$. Tienen efecto directo sobre la proliferación tumoral (a este respecto existen discrepancias entre los autores $)^{1}$, la angiogénesis y la expresión antigénica ${ }^{4}$. Los mecanismos por los que ejercen su acción antitumoral son aún desconocidos; no se sabe si en ensayos clínicos los tratamientos inmunomoduladores producen más respuestas o respuestas más duraderas que los brazos control no tratados. Muchos autores coinciden en que es esencial la activación por parte de estas citokinas de células $\mathrm{T}$ citotóxicas ${ }^{1}$ y natural-killers (NK). A este respecto, en algunos estudios los niveles previos de células $\mathrm{T}$ y NK se han mostrado predictores de respuesta al tratamiento con IFN- $\alpha$ e IL-2 en CRM, y también está descrita la relación entre la activación de linfocitos $\mathrm{T}$ en sangre periférica y la respuesta al tratamiento con estas citokinas ${ }^{4}$. Sin embargo en la mayoría de los pacientes la respuesta inmune al CR es insuficiente para controlar la enfermedad ${ }^{7}$. Dicha respuesta inmune conllevaría la unión del antígeno tumoral (epítope) al complejo mayor de histocom- patibilidad tipo I y II, la activación de células CD8+/CD4+ con producción de citokinas, el reclutamiento de otras células $\mathrm{T}$ efectoras y la producción de células $\mathrm{T}$ de memoria dirigidas a destruir la célula tumoral ${ }^{7}$. La evidencia que apoya la estrecha relación entre el CR y la respuesta inmune mediada por este tipo de células es numerosa. Así, células T clonales antígeno-específicas procedentes de infiltrados en tumores renales son capaces de producir lisis de células de CR in vitro ${ }^{7}$. Sin embargo, la mayoría son células T Helper CD4+ no citotóxicas que, cuando son activadas, son incapaces de secretar suficientes citokinas efectoras, por lo que son incapaces de causar lisis tumoral por sí solas?

Otros hallazgos ponen de manifiesto las complejas relaciones celulares y moleculares que se establecen entre el huesped y el tumor. En este sentido, el CR se caracteriza por la pérdida de actividad del gen supresor tumoral von Hippel Lindau ${ }^{58,59}$, pudiendo estar alteradas además varias rutas de señalización intracelulares (incluyendo NFאB y tirosin-kinasas específicas) ${ }^{7}$. También se ha publicado que células de CR avanzados pierden la expresión de alelos de HLA clase I, pudiendo evitar el ser reconocidas por células $\mathrm{T} \mathrm{CD} 8+{ }^{1,7}$. Una posible explicación a los casos de RE, es el hecho de que antígenos de superficie normales, habitualmente tolerados, se hacen más reactivos en presencia de células dendríticas presentadoras reclutadas en el contexto de procesos de necrosis. Dicha necrosis o destrucción tumoral, sea espontánea o inducida, puede montar una respuesta inflamatoria que provoque la regresión del tumor ${ }^{1}$.

Michael y Pandha, en una revisión publicada en 2003, identifican varios marcadores celulares y moleculares asociados al CRM, pero ninguno de ellos se mostró superior al estadio tumoral y al grado de diferenciación como factores pronósticos ${ }^{7}$. Estos marcadores se resumen en la Tabla I.

En esta misma revisión, la identificación de antígenos asociados al tumor, como por ejemplo RAGE1 -asociado a menos de un $2 \%$ de CR- o la proteína HLA-A2 mutada, junto con la especificidad de la respuesta inmune celular pueden llevar al desarrollo de nuevas estrategias terapéuticas. Entre ellas, las células T CD8+, aisladas del tumor primario, cultivadas in vitro y posteriormente re-infundidas en el paciente junto a IL-2 ${ }^{1,7}$ han dado lugar a respuestas muy variables (0-34\%) y en estudios fase III no se ha mostrado superior a IL-2 sola. 
TABLA I

\section{Marcadores celulares y Moleculares asociados a CRM}

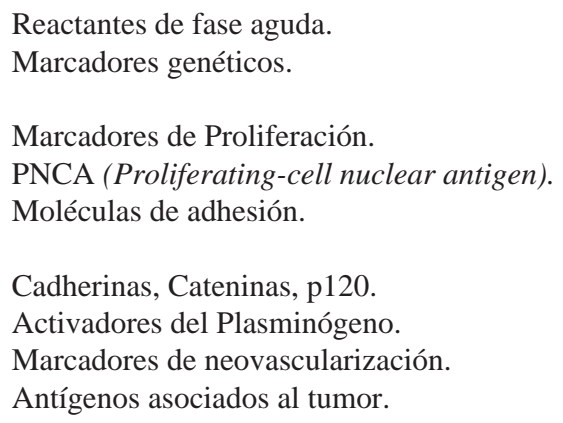

Reactantes de fase aguda.

Marcadores genéticos.

Marcadores de Proliferación.

PNCA (Proliferating-cell nuclear antigen).

Moléculas de adhesión.

Cadherinas, Cateninas, p120.

Activadores del Plasminógeno.

Marcadores de neovascularización.

Antígenos asociados al tumor.

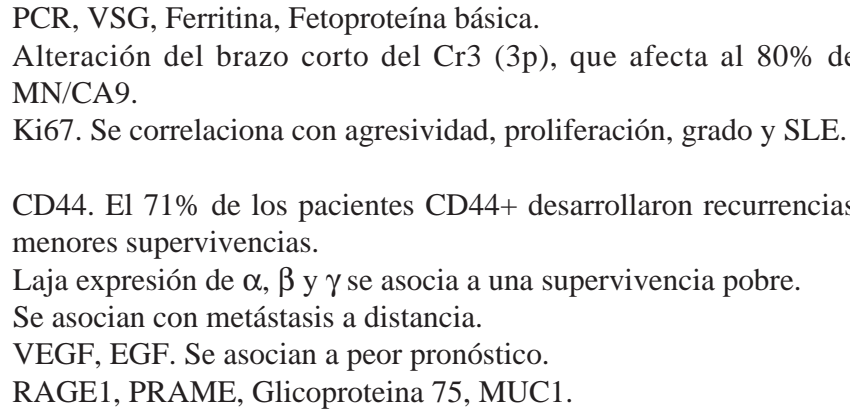

PCR, VSG, Ferritina, Fetoproteína básica. MN/CA9.

Ki67. Se correlaciona con agresividad, proliferación, grado y SLE. menores supervivencias.

Laja expresión de $\alpha, \beta$ y $\gamma$ se asocia a una supervivencia pobre.

Se asocian con metástasis a distancia.

VEGF, EGF. Se asocian a peor pronóstico.

RAGE1, PRAME, Glicoproteina 75, MUC1.

Alteración del brazo corto del Cr3 (3p), que afecta al $80 \%$ de los CR, Gen

CD44. El 71\% de los pacientes CD44+ desarrollaron recurrencias y presentaron

Tomado de: Michael A, Pandha HS. Renal-cell carcinoma: tumour markers, T-cell epitopes, and potential for new therapies. Lancet Oncol 2003;4:215-23.

Otra estrategia terapéutica importante en este sentido es la inmunoterapia dirigida con anticuerpos monoclonales (MAB). La constatación de la sobreexpresión del receptor para el factor de crecimiento epitelial (EGFR) en el CRM, cuya inmunoreactividad se asocia a mayor grado histológico y a peor supervivencia causa-específica ${ }^{54}$, ha conducido la investigación hacia el tratamiento con MAB contra dicho receptor (ABX-EGF), habiéndose demostrado respuestas in vitro ${ }^{7}$. Sin embargo, un estudio Fase II con Gefinitib (Inhibidor Tirosin-kinasa de EGFR) no ha mostrado efectos ${ }^{55}$.

La ya citada inactivación del gen von Hippel Lindau conlleva la sobreexpresión de factores de crecimiento pro-angiogénicos, en concreto el factor de crecimiento endotelial vascular (VEGF) y el factor de crecimiento derivado de plaquetas (PDGF) ${ }^{58}$. Recientemente se han desarrollado nuevos agentes contra estas dianas, en concreto el SU11248 (Sunitinib) y el AG013736, dos inhibidores moleculares orales con alta afinidad por la porción tirosin-kinasa de los receptores de VEGF y PDGF $^{58,59}$, con buen perfil de seguridad en estudios Fase $\mathrm{I}^{60}$ y resultados prometedores. Un estudio multicéntrico Fase II con Sunitinib sobre 63 pacientes con CR metastático y progresión tras una primera línea de inmunoterapia, consiguió un $40 \%$ de respuestas parciales y un $27 \%$ de estabilizaciones de la enfermedad de más de 3 meses $^{58}$. En este estudio la mediana de tiempo a la progresión fue de 8,7 meses y el tratamiento fue bien tolerado.

También se ha podido constatar la sobreexpresión de HER2/neu en un 20-30\% de los casos de CRM. Estos epítopes se procesan con HLA-A2 y para algunos investigadores son posibles dianas para vacunas de péptidos ${ }^{7}$. La radioinmunoterapia con MAB marcado con ${ }^{131}$ Iodo frente al antígeno G250, con expresión en el $75 \%$ de los $\mathrm{CR}$, ha confirmado su especificidad mediante estudios fase I y fase II $^{56-58}$. También cabe destacar las mutaciones puntuales de p53 (entre un 3 y un 33\% en CR) que generan nuevos epítopes susceptibles de constituirse en nuevas dianas? ${ }^{7}$.

Por último, dentro de estas nuevas opciones terapéuticas, las vacunas con células tumorales enteras irradiadas, péptidos tumorales, plásmidos-DNA o células dendríticas presentadoras de antígenos podrían tener un papel importante en el futuro del tratamiento del CRM.

Nuestro caso concreto se trata de una RE tipo 2 de Everson y Cole, tratado únicamente con nefrectomía ( $\sin$ IFN). La RE se objetivó 21 meses después de la nefrectomía y 12 meses tras la progresión. En la actualidad el paciente se encuentra asintomático y libre de enfermedad.

Correspondencia:

Dr. J. I. Rodríguez Melcón

Servicio de Oncología Radioterápica Hospital Universitario de Salamanca Paseo San Vicente, 58-182 E-37007 Salamanca nachorodriguezmelcon@hotmail.com 


\section{Bibliografía}

1. Young RC. Metastatic renal-cell carcinoma: what causes occasional dramatic regressions? N Engl J Med 1998;338(18):1305-6.

2. Michalsky JM. Kidney, Renal Pelvis, and Ureter. En: Perez CA, Brady LW, Halperin EC, Schmidt-Ullrich RK, editores. Principles and Practice of Radiation Oncology. Philadelphia: Lippincott Williams \& Wilkins; 2004. p. 1649-63.

3. De Riese W, Goldenberg K, Allhoff E, Stief C, Schlick R, Liedke S, et al. Metastatic renal cell carcinoma (RCC): spontaneous regression, long-term survival and late recurrence. Int Urol Nephrol 1991;23(1):13-25.

4. Glaspy JA. Therapeutic options in the management of renal cell carcinoma. Semin Oncol 2002;29(3 Suppl 7):41-6.

5. Van Poppel H, Baert L. Nephrectomy for metastatic renal cell carcinoma and surgery for distant metastases. Acta Urol Belg 1996;64(2):11-7.

6. Wagner JR, Merino MJ, Pass HI, Linehan WM, Walther MM. Pulmonary infarcts can mimic pulmonary metastases from renal cancer. J Urol 1997;158(5):1688-90.

7. Michael A, Pandha HS. Renal-cell carcinoma: tumour markers, T-cell epitopes, and potential for new therapies. Lancet Oncol 2003;4(4):215-23.

8. Kjaer M. The role of medroxyprogesterone acetate (MPA) in the treatment of renal adenocarcinoma. Cancer Treat Rev 1988;15(3):195-209.

9. Collichio FA, Pandya K. Interferon alpha-2b and megestrol acetate in the treatment of advanced renal cell carcinoma: a phase II study. Am J Clin Oncol 1998;21(2):209-11.

10. Ludwig G, Jentzsch R, Nuri M. Spontaneous regression of pulmonary metastases from hypernephroma: report of a case and review of literature (author's transl). Med Klin 1977;72(49):2118-21.

11. MacManus MP, Harte RJ, Stranex S. Spontaneous regression of metastatic renal cell carcinoma following palliative irradiation of the primary tumour. Ir J Med Sci 1994;163(10):461-3.

12. Motzer RJ, Carducci MA, Fishman M, Hancock SL, Hauke RJ, editors. Kidney Cancer - v.2.2005 [monograph on the internet]. Jenkintown, Philadelphia: NCCN Clinical Practice Guidelines in Oncology; 2005 [cited 2005 Apr 5]. Available from: http://www.nccn.org/professionals/physician_gls/PDF/kidney.pdf.

13. Yang JC, Haworth L, Sherry RM, Hwu P, Schwartzentruber DJ, Topalian SL, et al. A randomized trial of bevacizumab, an anti-vascular endothelial growth factor antibody, for metastatic renal cancer. N Engl J Med 2003;349(5): 427-34.

14. Chang KC, Chan KL, Lam CW. Spontaneous regression of renal cell carcinoma metastases. Hong Kong Med J 1999;5(1):72-5.

15. Crespy G. Spontaneous recovery from metastatic cancer of the kidney. Favourable role of Vitamin E? J Chir (Paris) 1993;130(11):470-4.

16. Czaplicki M, Malewski AW, Kuzaka B, Mayzner-Zawadz- ka E. The puzzle of spontaneous regression of pulmonary metastasis of renal carcinoma (after many years of observing the patient). Pol Tyg Lek 1993;48(20-22):485-7.

17. Davis SD, Koizumi JH, Pitts WR. Spontaneous regression of pulmonary metastases from renal cell carcinoma. Urology 1989;33(2):141-4.

18. De Riese W, Goldenberg K, Allhoff E, Jonas U. Spontaneous regression of metastatic renal carcinoma with longterm survival. Br J Urol 1991;68(1):98-100.

19. Guthbjartsson T, Gislason T. Spontaneous regression of brain metastasis secondary to renal cell carcinoma. Scand J Urol Nephrol 1995;29(2):215-7.

20. Hammad AM, Paris GR, van Heuven WA, Thompson IM, Fitzsimmons TD. Spontaneous regression of choroidal metastasis from renal cell carcinoma. Am J Ophthalmol 2003;135(6):911-3.

21. Hensiek AE, Kellerman AJ, Hill JT. Spontaneous regression of a solitary cerebral metastases in renal carcinoma followed by meningioma development under medroxyprogesterone acetate therapy. Br J Neurosurg 2000;14(4):3546.

22. Kallmeyer JC, Dittrich OC. Spontaneous regression of metastases in a case of bilateral renal cell carcinoma. J Urol 1992;148(1):138-40.

23. Lokich J. Spontaneous regression of metastatic renal cancer. Case report and literature review. Am J Clin Oncol 1997;20(4):416-8.

24. Mage P, Ballanger P, Lakdja F, Guibert JL, Vincent J, Chomy $\mathrm{P}$, et al. Spontaneous regression of pulmonary images interpreted as metastases of kidney cancer. Apropos of 2 cases. Ann Urol (Paris) 1986;20(4):271-4.

25. Marcus SG, Choyke PL, Reiter R, Jaffe GS, Alexander RB, Linehan WM, et al. Regression of metastatic renal cell carcinoma after cytoreductive nephrectomy. J Urol 1993;150(2 Pt 1):463-6.

26. Martinez-Pineiro L, de la Pena J, Picazo ML, Jimenez Leon J, Beneitez M, Martinez-Pineiro JA. Spontaneous regression of pulmonary metastases and long-term survival of a patient with metastatic renal cell carcinoma, after immunostimulation with bacillus Calmette-Guerin and extirpation of brain and contralateral lung metastases. Eur Urol 1988;15(1-2):146-9.

27. Mathias DB. A case of spontaneous regression of pulmonary metastases arising from hypernephroma following nephrectomy. Br J Urol 1971;43(1):65-8.

28. Mohr SJ, Whitesel JA. Spontaneous regression of renal cell carcinoma metastases after preoperative embolization of primary tumor and subsequent nephrectomy. Urology 1979;14(1):5-8.

29. Muller GA, Diem U, Fritz U, Walter E, Waller HD. Spontaneous remission of lung metastases in renal cell carcinoma. Dtsch Med Wochenschr 1989;114(11):420-3.

30. Nakano E, Sonoda T, Fujioka H, Okuyama A, Matsuda M, Osafune M, et al. Spontaneous regression of pulmonary metastases after nephrectomy for renal cell carcinoma. Eur Urol 1984;10(3):212-3.

31. Omland H, Fossa SD. Spontaneous regression of cerebral 
and pulmonary metastases in renal cell carcinoma. Scand J Urol Nephrol 1989;23(2):159-60.

32. Puchetti V. Spontaneous regression of pulmonary metastases of hypernephroma. Chir Ital 1966;18(6):1017-26.

33. Rossi A, Gobbi PG, Foresti G, D'Andrea F, Rutigliano L, Bertoloni D, et al. Spontaneous remission of visceral metastases of hypernephroma after nephrectomy. Description of a case and review of pathogenetic hypotheses. Recenti Prog Med 1991;82(11):591-7.

34. Skriver M, Nielsen JK. Spontaneous regression of pulmonary metastases after nephrectomy for renal cell carcinoma. Ugeskr Laeger 1995;157(44):6138-9.

35. Snow RM, Schellhammer PF. Spontaneous regression of metastatic renal cell carcinoma. Urology 1982;20(2):17781.

36. Stephenson HE, Jr., Delmez JA, Renden DI, Kimpton RS, Todd PC, Charron TL, et al. Host immunity and spontaneous regression of cancer evaluated by computerized data reduction study. Surg Gynecol Obstet 1971;133(4):649-55.

37. Thomas PJ, Stott M, Royle GT. Spontaneous regression of subcutaneous and pulmonary metastases from renal carcinoma. Br J Urol 1989;63(1):102-3.

38. Thoroddsen A, Gudbjartsson T, Geirsson G, Agnarsson BA, Magnusson K. Spontaneous regression of pleural metastases after nephrectomy for renal cell carcinoma-a histologically verified case with nine-year follow-up. Scand J Urol Nephrol 2002;36(5):396-8.

39. Vizel M, Oster MW, Austin JH. Spontaneous regression of a pulmonary metastasis after nephrectomy for renal cell carcinoma. J Surg Oncol 1979;12(2):175-80.

40. Vogelzang NJ, Priest ER, Borden L. Spontaneous regression of histologically proved pulmonary metastases from renal cell carcinoma: a case with 5-year followup. J Urol 1992;148(4):1247-8.

41. Wyczolkowski M, Klima W, Bieda W, Walas K. Spontaneous regression of hepatic metastases after nephrectomy and metastasectomy of renal cell carcinoma. Urol Int 2001;66(2):119-20.

42. Yoshino S, Wakui M. Spontaneous regression of lung metastasis of renal cell carcinoma: a case report. Hinyokika Kiyo 1988;34(12):2167-9.

43. Ridings GR. Renal adenocarcinoma: regression of pulmonary metastases following irradiation of primary tumor. Cancer 1971;27(4):936-8.

44. Bloom HJG, Payne PM. Hormone treatment of renal tumors: experimental and clinical observations. In: Riches E, editor. Tumours of the Kidney and Ureter. Edimburgh: E\&S Livingstone Ltd; 1964. p. 311.

45. Everson TC, Cole WH. Spontaneous Regression of Cancer. Philadelphia: W.B. Saunders, 1966.

46. Atmani A, Valleix D, Blaise S, Labrousse F, Pech de la Clause B, Lachachi F, et al. Intrathyroid metastases from kidney cancers: two case reports. Ann Chir 2002;127(7): 532-4.

47. Koodziejski L, Goralczyk J, Dyczek S, Duda K, Nabiaek $\mathrm{T}$. The role of surgery in lung metastases. Eur J Surg Oncol 1999;25(4):410-7.
48. Wronski M, Arbit E, Russo P, Galicich JH. Surgical resection of brain metastases from renal cell carcinoma in 50 patients. Urology 1996;47(2):187-93.

49. Garcia Tabar PJ, Montoya Lirola MD, Etxepare Arrosagaray P, Castillo Jimeno JM, Acinas Garcia O, Alvarez Martinez J. Spontaneous disappearance of pulmonary metastasis secondary to renal cell carcinoma after nephrectomy. Presentation of a case and review of the literature. Actas Urol Esp 1992;16(5):430-4.

50. Kerbl K, Pauer W. Spontaneous regression of osseous metastasis in renal cell carcinoma. Aust N Z J Surg 1993;63(11):901-3.

51. Deweerd JH, Hawthorne NJ, Adson MA. Regression of renal cell hepatic metastasis following removal of primary lesions. J Urol 1977;117(6):790-2.

52. Altschuler EL, Ray A. Spontaneous regression of a pancreatic metastasis of a renal cell carcinoma. Arch Fam Med 1998;7(6):516-7.

53. Pérez Romasanta LA, Calvo Manuel FA, editores. Guía terapéutica de soporte en Oncología Radioterápica. GICOR. Barcelona: Masson; 2004.

54. Uhlman DL, Nguyen P, Manivel JC, Zhang G, Hagen K, Fraley E, et al. Epidermal growth factor receptor and transforming growth factor alpha expression in papillary and nonpapillary renal cell carcinoma: correlation with metastatic behavior and prognosis. Clin Cancer Res 1995;1(8):913-20.

54. Druker B, et al. Phase II trial of ZD1839 (Iressa), an EGF receptor inhibitor, in patients with advanced renal cell carcinoma. Proc Am Assoc Clin Oncol 2002; 21: 181a (Abstr).

55. Divgi CR, Bander NH, Scott AM, O’Donoghue JA, Sgouros $\mathrm{G}$, Welt $\mathrm{S}$, et al. Phase I/II radioimmunotherapy trial with iodine-131-labeled monoclonal antibody G250 in metastatic renal cell carcinoma. Clin Cancer Res 1998;4(11): 2729-39.

56. Oosterwijk E, Bander NH, Divgi CR, Welt S, Wakka JC, Finn RD, et al. Antibody localization in human renal cell carcinoma: a phase I study of monoclonal antibody G250. J Clin Oncol 1993;11(4):738-50.

57. Uemura H, Beniers AJ, Okajima E, Debruyne FM, Oosterwijk E. Vaccination with anti-idiotype antibodies mimicking a renal cell carcinoma-associated antigen induces tumor immunity. Int J Cancer 1994;58(4):555-61.

58. Motzer RJ, Michaelson MD, Redman BG, Hudes GR, Wilding G, Figlin RA, et al. Activity of SU11248, a multitargeted inhibitor of vascular endothelial growth factor receptor and platelet-derived growth factor receptor, in patients with metastatic renal cell carcinoma. J Clin Oncol 2006; 24(1):16-24.

59. Rini BI. SU11248 and AG013736: current data and future trials in renal cell carcinoma. Clin Genitourin Cancer 2005;4(3):175-80.

60. Faivre S, Delbaldo C, Vera K, Robert C, Lozahic S, Lassau N, et al. Safety, pharmacokinetic, and antitumor activity of SU11248, a novel oral multitarget tyrosine kinase inhibitor, in patients with cancer. J Clin Oncol 2006; 24(1):25-35. 